\title{
Homogeneous Distances to Several Stellar Groups in the Second Galactic Quadrant
}

\author{
Nadia Kaltcheva $^{1}$, Kevin Moran ${ }^{1}$, Thomas Gehrman ${ }^{1}$, Valeri Golev ${ }^{2}$ \\ ${ }^{1}$ Department of Physics and Astronomy, University of Wisconsin Oshkosh, Oshkosh, USA \\ ${ }^{2}$ Department of Astronomy, Faculty of Physics, St. Kliment Ohridski University of Sofia, Sofia, Bulgaria \\ Email:kaltchev@uwosh.edu, valgol@phys.uni-sofia.bg \\ Received March 29, 2013; revised April 30, 2013; accepted May 8, 2013
}

Copyright (C) 2013 Nadia Kaltcheva et al. This is an open access article distributed under the Creative Commons Attribution License, which permits unrestricted use, distribution, and reproduction in any medium, provided the original work is properly cited.

\begin{abstract}
A photometric study in the $u v b y \beta$ system of a $20^{\circ} \times 20^{\circ}$ field in direction of the Cas OB6 and Per OB1 associations is presented. All currently available $u v b y \beta$ photoelectric data are used to obtain homogeneous color excesses and distances of nearly 230 stars of spectral types O-B9. The double cluster h \& $\chi$ Per, NGC 663 and NGC 1502 are well represented in our sample. The sample also contains the brightest members of the young open clusters IC 1805, IC 1848, St 2, St 7 and ASCC9. We found that, within the errors, $h \& \chi$ Per, NGC 663, IC 1805, IC 1848 and ASCC9, together with the Per OB1 association are located at very similar distance moduli between 11.0 to 11.3 mag. Our results indicate that the distance spread among these objects is less than the previously estimated, suggested that they could represent starforming complexes located at the same distance.
\end{abstract}

Keywords: Open Clusters and Associations: Individual (NGC 869); Open Clusters and Associations: Individual (NGC 884); Open Clusters and Associations: Individual (Per OB1); Open Clusters and Associations: Individual (NGC 663); Open Clusters and Associations: Individual (NGC 1502)

\section{Introduction}

The central part of the second Galactic quadrant, located between $125^{\circ}$ and $145^{\circ}$ galactic longitude and $-10^{\circ}$ and $10^{\circ}$ galactic latitude contains several isolated HII regions. Among them, Sh 2-190 and Sh 2-199 are the most prominent, found toward $1=135^{\circ}$ in the disk. Several very young clusters (IC 1805, 1848 and Col 33) are associated with these nebulae. The sparse Cas OB6 association, which may be part of the even more extended Cam OB1 is also in this direction. The relatively compact Per OB1, surrounding the double cluster $\mathrm{h}$ and $\chi$ Per, is located several degrees below the disk. Other clusters, among which NGC 663 are found in an area free of $\mathrm{H} \alpha$ emission toward $1=130^{\circ}$. The field presents a unique opportunity to study possible co-evolution of stellar clusters and associations. Some of the populated clusters, especially $\mathrm{h}$ and $\chi$ Per, have been extensively investigated both in terms of broad-band (UBV, VI) and intermediate-band $(u v b y \beta)$ photometries.

The purpose of this paper is to provide an overview of the structure of the field based on homogeneous distance and reddening estimates for as many young stars as possible. For our purpose we utilize the $u v b y \beta$ system (Strö- mgren [1], Crawford \& Mander [2]) which is arguably better suited to the study of individual stars in terms of stellar luminosity than any other system in wide use. The $u v b y \beta$ photometry-derived parameters allow reliable distance determinations, thereby helping to distinguish between closely spaced groups lying along the line of sight. It should be also noted that the distance determination in the $u v b y \beta$ system, when based on early-type stars, is metallicity independent. This helps to reduce the uncertainties in the cluster distances in comparison to those obtained via main-sequence (MS) fitting, where an assumption regarding the metallicity is necessary.

This is the first article of a series of papers dedicated to the structure of the second Galactic quadrant, as delineated by open clusters and $\mathrm{OB}$ associations for which homogeneous $u v b y \beta$ photometric distances can be derived.

\section{The Sample}

All $u v b y \beta$ data within the studied coordinate range was extracted from the Hauck \& Mermilliod catalog [3]. The sample contains 226 stars of spectral types O to B9 with complete $u v b y \beta$ photometry. Figure 1 presents these 
stars overplotted on a $\mathrm{H} \alpha$ map (smoothed to 4 arcmin resolution to remove star residuals, Gaustad et al. [4]), obtained via the SkyView VO interface (McGlynn et al. [5]).

\section{Calculation of Interstellar Extinction and Distances}

In order to infer stellar parameters from the photoelectric photometry, we follow the procedure described in detail by Kaltcheva \& Hilditch [6]. The spectral and luminosity classifications (MKK) were extracted from the SIMBAD database and used in conjunction with the classification $\left[\mathrm{c}_{1}\right]$ vs. $\left[\mathrm{m}_{1}\right]$ diagram (Strömgren [1], not shown here). Only stars with $\left[\mathrm{m}_{1}\right]$ smaller than 0.15 are used in the following analysis, since photometrically they should be earlier types than B9. We found a good agreement between the photometric classification and the MKK types. Note that in the $u v b y \beta$ system both the color excess $\mathrm{E}(\mathrm{b}-\mathrm{y})$ and absolute magnitude $\left(\mathrm{M}_{\mathrm{V}}\right)$ calculations do not rely on a precise determination of the spectral subtype, since the calculations are carried on in the same

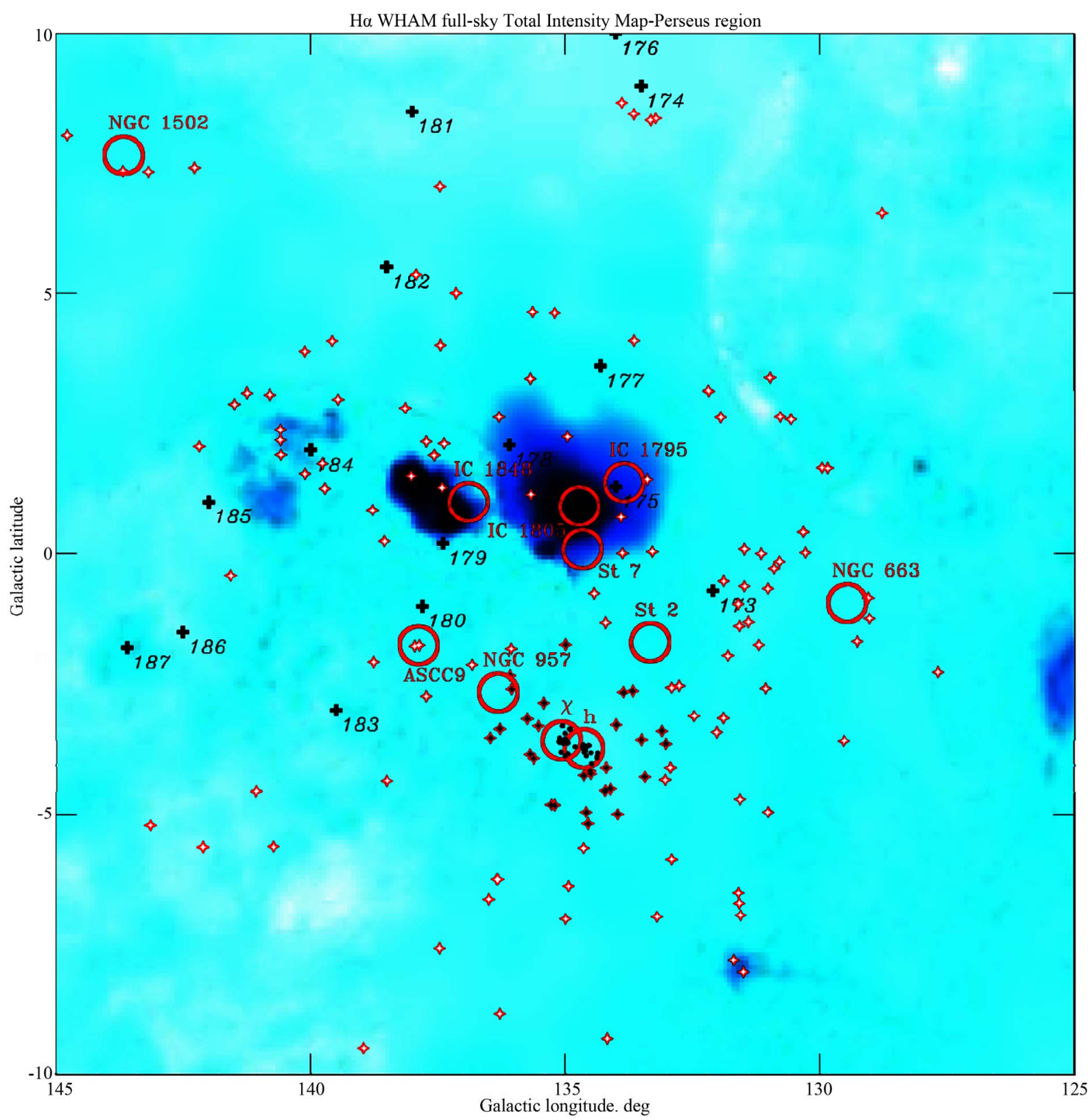

Figure 1. All stars in the field with $u v b y \beta$ photometry available in the Hauck \& Mermilliod catalog [3] plotted in galactic coordinates (longitude and latitude, in degrees). The stars are superimposed on a H $\alpha$ obtained via the SkyView VO interface. The open clusters with $u v b y \beta$ photometry are shown with large circles and are labeled. The star-forming complexes by Russeil [26], also labeled, are marked with plus symbols. 
way for all stars in a given spectral range (e.g. O-B9), depending only on the luminosity class (LC). This reduces the errors when calculating these parameters in comparison to the spectrophotometric method.

We used the Crawford's [7] calibration to obtain the color excesses for LC III, IV and V. The calibration by Kilkenny \& Whittet [8] was used for LC II, Ib, Iab and Ia. We adopted $\mathrm{R}=3.18$ and $\mathrm{E}(\mathrm{B}-\mathrm{V})=\mathrm{E}(\mathrm{b}-\mathrm{y}) / 0.74$ to obtain $\mathrm{V}_{0}$. The calibration by Balona \& Shobbrook [9] was utilized for all stars to derive the Mv values. Since we are dealing with early spectral types, the presence of emission lines in the stellar spectra is the largest source of error in the calculated absolute magnitudes. However, the $\beta$ vs. $\mathrm{c}_{0}$ diagram (not shown here) reveals that very few stars deviate from the main sequence and have photometry affected by emission. Note that the outlined procedure provides photometric distances in excellent agreement with the recalculated Hipparcos data (Kaltcheva \& Makarov [10]). In addition, the Balona \& Shobbrook [9] luminosity calibration has been tested via the Hipparcos data and shown to be reliable (Kaltcheva \& Knude [11], Torra et al. [12], Kaltcheva \& Golev [13]). The expected uncertainties in $\mathrm{M}_{\mathrm{V}}$ are of the order of \pm 0.3 mag for $\mathrm{O}$ and B types of LC III-V, and \pm 0.5 mag for B-type supergiants. An uncertainty of \pm 0.3 mag in $\mathrm{M}_{\mathrm{V}}$ propagates to an asymmetric error of $-13 \%$ to $+15 \%$, and uncertainties of \pm 0.5 mag result in $-21 \%$ to $+26 \%$ error in the derived distances.

\section{Photometry-Derived Results}

The derived stellar parameters for the sample stars are available from the authors upon request. The diagrams color excess $\mathrm{E}(\mathrm{b}-\mathrm{y})$ vs. distance moduli $(\mathrm{DM}), \mathrm{V}_{0}$ vs. $(b-y)_{0}$ and $M_{V}$ vs. $(b-y)_{0}$ were examined to reveal spatially coherent structures in the studied longitude range.

\section{1. $h$ and $\chi$ Per and Per OB1}

The first extensive $u v b y \beta$ photometry of $\mathrm{h}$ and $\chi$ Per (NGC 869 and NGC 884, respectively) was performed by Crawford et al. [14]), who concluded that both clusters have nearly the same age and distance, the distance modulus being $11.4 \pm 0.4$ mag. Balona \& Shobbrook [9] corrected this value for evolutionary effects and adopted a distance modulus of 11.16 for both clusters. Marco \& Bernabeu [15] presented $u v b y \beta$ CCD photometry of nearly 350 stars in the area of the cluster, to a limiting magnitude $\mathrm{V}=16.5$. From ZAMS fitting, they derived a distance moduli of $11.66 \pm 0.20$ and $11.56 \pm 0.20$ for $\mathrm{h}$ Per and $\chi$ Per, respectively, and adopted $11.60 \pm 0.20$ to the double cluster. Based also on $u v b y \beta$ CCD photometry, Capilla \& Fabregat [16] found that both clusters share a common distance modulus of $11.7 \pm 0.1 \mathrm{mag}$. However, analyzing $u v b y \beta$ literature data, Malysheva [17] obtained different distances to the clusters ( $2025 \mathrm{pc}$ to NGC 869 and 2426 pc to NGC 884-distance moduli 11.53 and 11.92 mag respectively).

The findings based on broad-band photometry seem to be a little bit more controversial. Based on CCD UBV data and spectroscopic parallaxes Slesnick et al. [18] obtained a distance modulus of $12.5 \pm 0.5$ mag for objects within the cluster cores, but including only stars brighter than $\mathrm{V}=10.5 \mathrm{mag}$ yielded $11.95 \pm 0.2$. Their best-fitting distance modulus provides $11.85 \pm 0.05 \mathrm{mag}$, corresponding to a distance of $2344 \pm 54 \mathrm{pc}$. Currie et al. [19] presented very extensive VI photometry and spectroscopy of $\mathrm{h} \& \chi$ Per down to sub-solar masses. They found both clusters, as well as the halo, to have similar color excesses $(\mathrm{E}(\mathrm{B}-\mathrm{V})=0.52-0.55)$ and distance moduli $(\mathrm{DM}=11.8-11.85$ (for $\mathrm{R}=3.12)$ ). These estimates lead to a distance of $2300-2350 \mathrm{pc}$. The most recent catalog of open clusters Kharchenko et al. [20] lists a distance of 2079 pc to NGC 869 and 2345 pc to NGC 884, estimates currently accepted in the WEBDA database. Selected values of distance modulus, age and reddening taken from the literature have been recently systematized by Southworth et al. [21] and also by Currie et al. [19]. In general, some authors claim that h Per (NGC 869) is closer and younger than $\chi$ Per (NGC 884), while other state that the clusters have similar distance and age.

The double cluster is sometimes considered to be the core of the Per OB1 association. Humphreys [22] identified 105 members of the association within longitude range from $132^{\circ}$ to $136^{\circ}$ and latitude from $-5^{\circ}$ to $-2.5^{\circ}$, at a distance modulus $11.83 \pm 0.06$. Using the cluster-fitting method, Garmany \& Stencel [23] derived a distance modulus of 11.8 for related members. The Per OB1 members identified by the latter authors span a larger range in Galactic longitude $\left(131^{\circ}\right.$ to $\left.139^{\circ}\right)$ and Galactic latitude $\left(-6.5^{\circ}\right.$ to $\left.0^{\circ}\right)$. It is a long-standing question whether or not the double cluster is spatially connected to Per OB1 (see for example the discussion in Slesnick et al. [18]). Although containing more than 10 O-type stars, Per OB1 is not associated with any known HII regions or giant molecular cloud (see Lee \& Lim [24] for a thorough discussion).

In a $3^{\circ} \times 3^{\circ}$ field toward the cluster, there are $82 \mathrm{O}$ and B-type stars with photometry available in the Hauck \& Mermilliod catalog [3]. It should be mentioned that for $h$ and $\chi$ Per and Per OB1 the available $u v b y \beta$ photometry of the vast majority of stars comes from a single source, namely Crawford et al. [14] and is thus very homogeneous. The diagrams color excess $\mathrm{E}(\mathrm{b}-\mathrm{y})$ vs. Distance Modulus, $\mathrm{V}_{0}$ vs. $(\mathrm{b}-\mathrm{y})_{0}$ and $\mathrm{M}_{\mathrm{V}}$ vs. $(\mathrm{b}-\mathrm{y})_{0}$ are presented in Figure 2. The stars are shown with different symbols as follows: open squares for NGC 869; open cirles for NGC 884; filled symbols for the stars of the sur- 

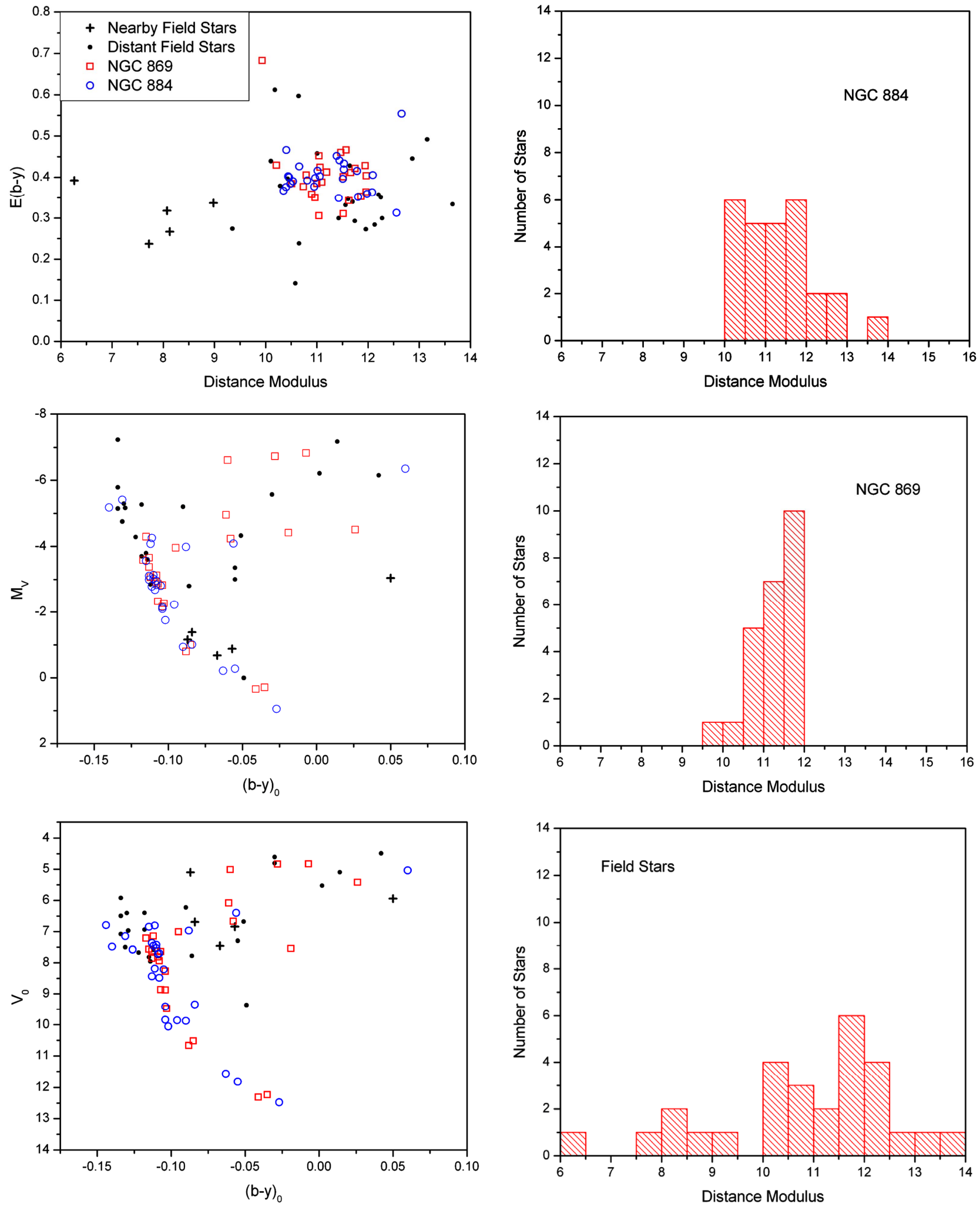

Figure 2. All O-B9 stars with calculated distances and color excesses in the sample in the field of Per OB1 association. (Left) The diagrams color excess $E(b-y)$ vs. Distance Modulus, $V_{0}$ vs. $(b-y)_{0}$ and $M_{V} v s$. $(b-y)_{0}$. The cluster stars and the field stars are plotted with different symbols as follows: open squares: NGC 869; open circles: NGC 884; plus symbols: nearby field stars; filled symbols: distant field stars. (Right) The histograms of the individual distances of NGC 869, NGC 884 and the field stars. 
rounding Per OB1 association; plus symbols for the several nearby field stars. The average distance moduli and color excesses for NGC 869, NGC 884 and Per OB1 are presented in the first part of Table 1. According to this sample, the clusters are located at the same distance (we adopt $11.23 \pm 0.6$ s.d.) and have similar excess $(\mathrm{E}(\mathrm{b}-\mathrm{y})$ $=0.40 \pm 0.05$ ). These results are consistent with both following Balona \& Shobbrook [9] and Crawford et al. [14]). The estimated distance to Per OB1 is similar to the distance to the clusters, although a larger spread of the individual distance moduli can be seen, as expected for an $\mathrm{OB}$ association. However, the average distance to the association is more sensitive to the completeness of the sample, in comparison to the average distances of the clusters. The $\mathrm{V}_{0}$ vs. $(\mathrm{b}-\mathrm{y})_{0}$ and $\mathrm{M}_{\mathrm{V}}$ vs. $(\mathrm{b}-\mathrm{y})_{0}$ diagrams are also indicative for a similar distance and, to a large extent, stellar content for both the clusters and the surrounding stars. It could be noticed, that NGC 869 contains nearly three times more evolved stars in comparison to NGC 884, but is difficult to evaluate whether this is a selection effect.

In addition to the sample described above, we also considered the two CCD $u v b y \beta$ data-sets available at present for NGC 869 and NGC 884 (Marco \& Bernabeu [15], Capilla \& Fabregat [16]). The estimates of distance moduli and color excesses that we adopt based on these samples are shown in the second and third part of Table 1. Using all early-type stars in the data-set of Marco \& Bernabeu [15] yields distance moduli of $11.91( \pm 0.64$ s.d., \pm 0.06 s.e.) for NGC 869 (123 stars) and 12.00 ( \pm 0.36 s.d., \pm 0.04 s.e.) for NGC 884 (93 stars). For NGC 869 the majority of these stars are located between distance moduli 10.5 and 12.5 . If we use only them, the average value is 11.78 ( \pm 0.44 s.d., \pm 0.04 s.e. $)$ and this is the estimate we adopt in Table 1. For NGC 884 the majority of the stars are located between distance moduli 11.5 and 12.5. If we use only these stars, the average value is 12.00 ( \pm 0.23 s.d., \pm 0.03 s.e.) (the one listed in Table 1). The data-set of Capilla \& Fabregat [16] yields DM = 11.60 ( \pm 0.42 s.d., \pm 0.04 s.e.) (NGC 869,99 stars with $\mathrm{DM}$ between 10.5 and $12.5 \mathrm{mag})$ and $11.71( \pm 0.58$ s.d., \pm 0.06 s.e) (NGC 884, 91 stars with DM between 10.5 and $12.0 \mathrm{mag}$ ). Capilla \& Fabregat [16] obtained 11.5 and 11.9 to the clusters based on cluster-fitting method. The agreement between our and their results, which are based on different methods, is very good.

It is obvious that all available $u v b y \beta$ data-sets yield to the conclusion that the clusters are located at similar distances. The CCD data provide however distance moduli up to 0.75 mag larger in comparison to the photoelectric photometry. In general, the photoelectric photometry (like the one from Crawford et al. [14]) has been shown to be superior to CCD $u v b y \beta$ photometry (see, for example, $[25])$. The $\left[\mathrm{c}_{1}\right]$ vs. $\left[\mathrm{m}_{1}\right]$ diagram, based on photoelectric data shows a much tighter main sequence in comparison to the corresponding diagrams utilizing CCD data (not shown here). In general, it is known that lower precision of the photometry can affect the accuracy of the derived stellar parameters.

Regarding the color excess, the data-set of Marco \& Bernabeu [15] yields $\mathrm{E}(\mathrm{b}-\mathrm{y})=0.44( \pm 0.067$ s.d., \pm 0.006 s.e. $)$ and $\mathrm{E}(\mathrm{b}-\mathrm{y})=0.40( \pm 0.10$ s.d., \pm 0.001 s.e. $)$ for NGC 869 and NGC 884, respectively. The data-set of Capilla \& Fabregat [16] provides $\mathrm{E}(\mathrm{b}-\mathrm{y})$ of 0.44 ( \pm 0.069 s.d., \pm 0.006 s.e.) (NGC 869$)$ and 0.41 ( \pm 0.047 s.d., \pm 0.005 s.e) (NGC 884 ). Within the errors, all available $u v b y \beta$ data-sets lead to similar estimates of the color excess of both clusters. The estimates of the color excess do not change if stars with deviating distance moduli were excluded. We accept $\mathrm{E}(\mathrm{b}-\mathrm{y})=0.40( \pm 0.06$ s.d. $)$,

Table 1. Distance moduli and color excess for NGC 869, NGC 884 and field stars (Per OB1) based on the three uvby $\beta$ datasets available.

\begin{tabular}{ccccc}
\hline Quantity & NGC 869 & NGC 884 & Per OB1 & Source of photometry \\
\hline DM & $11.22 \pm 0.55 \pm 0.11$ & $11.24 \pm 0.69 \pm 0.14$ & $11.27 \pm 0.91 \pm 0.20$ & {$[3]$} \\
E (b-y) & $0.40 \pm 0.07 \pm 0.01$ & $0.40 \pm 0.05 \pm 0.01$ & $0.37 \pm 0.11 \pm 0.02$ & 21 \\
Number of stars & 24 & 26 & - & - \\
DM & $11.78 \pm 0.44 \pm 0.04$ & $12.00 \pm 0.23 \pm 0.03$ & - & {$[15]$} \\
E (b-y) & $0.44 \pm 0.03 \pm 0.003$ & $0.40 \pm 0.10 \pm 0.01$ & - & {$[16]$} \\
Number of stars & 104 & 66 & - \\
DM & $11.60 \pm 0.42 \pm 0.04$ & $11.71 \pm 0.58 \pm 0.06$ & - \\
E(b-y) & $0.44 \pm 0.07 \pm 0.01$ & $0.41 \pm 0.05 \pm 0.01$ & 91 & \\
Number of stars & 99 & 9 &
\end{tabular}

Note: For all estimates both the standard deviation and standard error of the mean are provided. The number of stars on which the estimates are based is shown. 
corresponding to $\mathrm{E}(\mathrm{B}-\mathrm{V})=0.54( \pm 0.08$ s.d.) mag.

It is worth noting that Marco \& Bernabeu [15] also provide a calculation of the average distance to the clusters based on the individual distance moduli of the Btype members. They calculate the intrinsic photometric indices adopting the average reddening for each cluster, and then for each star derive $\mathrm{M}_{\mathrm{V}}$ from $\mathrm{c}_{0}$ and $\beta$ following Balona \& Shobbrook [9] and use them to calculate the distance modulus for each cluster as the average of its members. For h Per (NGC 869), their procedure provides an average distance modulus of $11.4 \pm 0.5$, while the average value for $\chi$ Per (NGC 884) is $12.1 \pm 0.2$ and the clusters appear to be located at slightly different distances. Our procedure is different from the one used by Marco \& Bernabeu [15], since we do not use the average reddening of the clusters, but calculate the reddening for each star. It should be mentioned that the ZAMS-fitting for the data-set of Marco \& Bernabeu [15] (11.66 \pm 0.20 and $11.56 \pm 0.20$ for $\mathrm{h}$ Per and $\chi$ Per, respectively) provides slightly different distance moduli in comparison to the average values.

\subsection{The Field of Cas OB6 Association}

The relatively sparse Cas OB6 association is located towards the prominent HII regions Sh 2-190 and Sh 2-199. Humphreys [22] lists 27 O-B1 stars as members of the association, located between $1=\left(133^{\circ}, 138^{\circ}\right)$ and $\mathrm{b}=$ $\left(-0.3^{\circ}, 3^{\circ}\right)$ at $\mathrm{DM}=11.7$. Eight of these stars are members of the open cluster NGC 1805. Garmany \& Stencel [23] obtained a distance modulus of $11.9 \mathrm{mag}$ to the Cas OB6 association. In our sample there are 27 stars in this direction which are located significantly closer than the previous estimates-at $\mathrm{DM}=8.5( \pm 0.99$ s.d., 0.19 s.e. $)$. The stars are of relatively later spectral types (B2-B8) and apparently form a layer foreground to Cas OB6. Several very young clusters are found in this direction, IC 1805, IC 1848 and ASCC9, at DM 11.13, 11.2 and 10.97 , respectively. These are the most distant and youngest clusters in our sample. They are located within several adjacent star-forming complexes $(175,176$ - 180) as defined by Russeil [26]. The kinematic distances attached to these complexes show a large spread, while the distances obtained here for objects associated with the complexes are very similar.

Several other clusters with $u v b y \beta$ photometry are available in the field and are listed in Table 2 . NGC 1502 may be connected to the Cam OB1 association at $\mathrm{DM}=10$ (Humphreys [22]). For this cluster we obtained $\mathrm{DM}=9.83$. The rest of the clusters are foreground, located in a DM range of 6.6 to10 mag.

\section{Concluding Remarks}

We present a $u v b y \beta$ photometric investigation of a $20^{\circ} \times$ $20^{\circ}$ field in the second Galactic quadrant centered at coordinates $1, \mathrm{~b}=\left(135^{\circ}, 0^{\circ}\right)$. We use photoelectric data extracted from the Hauck \& Mermilliod catalog [3], and also CCD data available in the literature. Our study is restricted to stars earlier than $\mathrm{B} 9$ spectral type. We derive the individual color excess and distance modulus for each star and then calculate the average values for the clusters and associations represented in our sample.

We estimate a distance modulus of 11.23 ( \pm 0.6 s.d., \pm 0.12 s.e.), corresponding to a distance of $1762_{-95}^{+100}$ to $\mathrm{h}$ $\& \chi$ Per. The layer of field stars representative for the Per

Table 2. Derived parameters for the cluster sample.

\begin{tabular}{|c|c|c|c|c|c|c|c|c|}
\hline Cluster & $\mathrm{N}$ & $1^{\circ}$ & $\mathrm{b}^{\circ}$ & DM (sd; se) & $\mathrm{DM}[27]$ & $\Delta \mathrm{DM}$ & Age [27] & $\mathrm{E}(\mathrm{b}-\mathrm{y})(\mathrm{sd} ; \mathrm{se})$ \\
\hline IC 1795 & 1 & 133.845 & 1.376 & $7.61::$ & - & - & - & 0.423 \\
\hline NGC 957 & 1 & 136.319 & -2.656 & $9.81::$ & 11.71 & -1.90 & 7 & 0.596 \\
\hline IC 1848 & 4 & 136.898 & 1.0002 & $11.02(0.90 ; 0.45)$ & 11.51 & -0.49 & 6.84 & $0.47(0.02 ; 0.01)$ \\
\hline ASCC9 & 2 & 137.878 & -1.752 & $10.97:(0.44 ; 0.31)$ & 12.31 & -1.34 & 6.79 & $0.59(0.03 ; 0.02)$ \\
\hline NGC 1502 & 22 & 143.672 & 7.658 & $9.83(0.38 ; 0.08)$ & 10.00 & -0.17 & 7 & $0.55(0.03 ; 0.01)$ \\
\hline NGC 663 & 27 & 129.467 & -0.941 & $10.57(0.64 ; 0.12)$ & 11.92 & -1.35 & 7.4 & $0.59(0.06 ; 0.01)$ \\
\hline NGC 869 & 24 & 134.632 & -3.741 & $11.23(0.55 ; 0.11)$ & 11.59 & -0.36 & 7.069 & 0.54 \\
\hline NGC 884 & 26 & 135.066 & -3.588 & $11.23(0.69 ; 0.14)$ & 12.34 & -1.10 & 7.1 & 0.54 \\
\hline St 2 & 4 & 133.334 & -1.694 & $6.61(0.26 ; 0.13)$ & 7.41 & -0.80 & 8.23 & $0.24(0.09 ; 0.04)$ \\
\hline
\end{tabular}

Note: The object's name, number of stars with $u v b y \beta$ data and the galactic coordinates are given in the first four columns. The obtained here true distance moduli (together with the standard deviation and standard error of the mean provided), the distance moduli from the catalog of Dias et al. [27] and the difference between them are listed in columns 5,6 and 7. The age (in log t) adopted from Dias et al. [27] and the color excesses obtained here are listed in the last two columns. 
OB1 association is located at that same average distance. The average color excess is the same for all three groups. Evolved B-type stars are present in both clusters and among the field stars as well, but there are more numerous in NGC 869. Based on the $\mathrm{V}_{0}$ vs. $(\mathrm{b}-\mathrm{y})_{0}$ and $\mathrm{M}_{\mathrm{V}} \mathrm{vs}$. (b $-\mathrm{y})_{0}$ diagrams one might conclude that NGC 869, NGC 884, and Per OB1 are equally old, but no quantitative comparisons to isochrones were attempted due to the relatively small sample. Apparently, a slight controversy exists between the distance modulus to $\mathrm{h} \& \chi$ Per derived based on CCD and photoelectric data. Since the photoelectric $u v b y \beta$ photometry should be considered superior to $\mathrm{CCD} u v b y \beta$ photometry in terms of accuracy, preference should be given to the results based on photoelectric data.

According to our findings, the double cluster and the Per OB1 association are exactly at the same distance as the brightest members of the young groups IC 1805, IC 1848 and ASCC9. All of them are located in a $7^{\circ} \times 7^{\circ}$ area, containing the regions of intense HII emission in Sh 2-190 and Sh 2-199. IC 1805 may be part of Cas OB6 at an adopted distance modulus 11.7 (Humphreys [22]). This is an indication that the star-forming complexes associated with these objects may be also located at similar distances and clearly warrant a further investigation.

\section{Acknowledgements}

This work is supported by the National Science Foundation grant AST-0708950. N.K. acknowledges support from the University of Wisconsin Oshkosh Faculty Development Grant FDR705. K.M. acknowledges support from the Wisconsin Space Grant Consortium. This research has made use of the SIMBAD database, operated at CDS, Strasbourg, France.

\section{REFERENCES}

[1] B. Strömgren, "Spectral Classification through PhotoElectric Narrow-Band Photometry," Annual Review of Astronomy and Astrophysics, Vol. 4, 1966, pp. 433-473. doi:10.1146/annurev.aa.04.090166.002245

[2] D. L. Crawford and J. Mander, "Standard Stars for Photoelectric $\mathrm{H} \beta$ Photometry," The Astronomical Journal, Vol. 71, 1966, pp. 114-118. doi:10.1086/109865

[3] B. Hauck and M. Mermilliod, " $u v b y \beta$ Photoelectric Photometric Catalogue," Astronomy and Astrophysics Supplement Series, Vol. 129, No. 3, 1998, pp. 431-433. doi:10.1051/aas:1998195

[4] J. E. Gaustad, P. R. McCullough, W. Rosing and D. Van Buren, "A Robotic Wide-Angle H $\alpha$ Survey of the Southern Sky," Publications of the Astronomical Society of the Pacific, Vol. 113, No. 789, 2001, pp. 1326-1348. doi:10.1086/323969

[5] T. McGlynn, K. Scollick and N. White, "SkyView: The Multi-Wavelength Sky on the Internet," Proceedings of the 179th Symposium of the International Astronomical Union, Baltimore, 26-30 August 1996, pp. 465-466.

[6] N. T. Kaltcheva and R. W. Hilditch, "The Distribution of Bright OB Stars in the Canis Major-Puppis-Vela Region of the Milky Way," Monthly Notices of the Royal Astronomical Society, Vol. 312, No. 4, 2000, pp. 753-768. doi:10.1046/j.1365-8711.2000.03170.x

[7] D. L. Crawford, "Empirical Calibrations of the $u v b y, \beta$ Systems. II. The B-Type Stars," Astronomical Journal, Vol. 83, No. 1, 1978, pp. 48-63. doi:10.1086/112176

[8] D. Kilkenny and D. C. B. Whittet, "On the Intrinsic $u b v y$ Colours of Early-Type Supergiants," Monthly Notices of the Royal Astronomical Society, Vol. 216, 1985, pp. 127137.

[9] L. A. Balona and R. R. Shobbrook, "A Re-Calibration of the Luminosities of Early-Type Stars-Its Effect on the Cepheid Luminosity Scale," Monthly Notices of the Royal Astronomical Society, Vol. 211, 1984, pp. 375-390.

[10] N. Kaltcheva and V. Makarov, "The Structure and the Distance of Collinder 121 from Hipparcos and Photometry: Resolving the Discrepancy," The Astrophysical Journal Letters, Vol. 667, No. 2, pp. L155-L157.

[11] N. Kaltcheva and J. Knude, "A Test of B-Type Star $H \beta$ Photometric Distances via the HIPPARCOS Parallaxes," Astronomy and Astrophysics, Vol. 337, 1998, pp. 178182.

[12] J. Torra, D. Fernández and F. Figueras, "Kinematics of Young Stars. I. Local Irregularities," Astronomy and Astrophysics, Vol. 359, 2000, pp. 82-102.

[13] N. Kaltcheva and V. Golev, "Improved Distances to Several Galactic OB Associations," Stellar Clusters and Associations: A RIA workshop on GAIA, Granada, 23-27 May 2011, pp. 299-303. http://sca.iaa.es/content/proceedings

[14] D. L. Crawford, J. W. Glaspey and C. L. Perry, "FourColor and $\mathrm{H} \beta$ Photometry of Open Clusters. IV. $\mathrm{h}$ and $\chi$ Persei," The Astronomical Journal, Vol. 75, 1970, pp. 822-830. doi:10.1086/111027

[15] A. Marco and G. Bernabeu, "Photometric Study of the Double Cluster $\mathrm{h}$ and $\chi$ Persei," Astronomy and Astrophysics, Vol. 372, No. 2, 2001, pp. 477-494. doi:10.1051/0004-6361:20010441

[16] G. Capilla and J. Fabregat, "CCD $u v b y \beta$ Photometry of Young Open Clusters. I. The Double Cluster $\mathrm{h}$ and $\chi$ Persei," Astronomy and Astrophysics, Vol. 394, No. 2, 2002, pp. 479-488. doi:10.1051/0004-6361:20021200

[17] L. K. Malysheva, "Parameters of Open Clusters from uvby $\beta$ Photometry," Astronomy Letters, Vol. 23, No. 5, 1997, pp. 585-590.

[18] C. L. Slesnick, L. A. Hillenbrand and Ph. Massey, "The Star Formation History and Mass Function of the Double Cluster h and $\chi$ Persei," The Astrophysical Journal, Vol. 576, No. 2, 2002, pp. 880-893. doi:10.1086/341865

[19] T. Currie, J. Hernandez, J. Irwin, S. J. Kenyon, S. Tokarz, Z. Balog, A. Bragg, P. Berlind and M. Calkins, "The Stellar Population of $\mathrm{h}$ and $\chi$ Persei: Cluster Properties, Membership, and the Intrinsic Colors and Temperatures of Stars," The Astrophysical Journal Supplement, Vol. 
186, No. 2, 2010, pp. 191-221. doi:10.1088/0067-0049/186/2/191

[20] N. V. Kharchenko, A. E. Piskunov, S. Röser, E. Schilbach and R.-D. Scholz, "Astrophysical Parameters of Galactic Open Clusters," Astronomy and Astrophysics, Vol. 438, No. 3, 2005, pp. 1163-1173. doi:10.1051/0004-6361:20042523

[21] J. Southworth, P. F. L. Maxted and B. Smalley, "Eclipsing Binaries in Open Clusters-I. V615 Per and V618 Per in h Persei," Monthly Notices of the Royal Astronomical Society, Vol. 349, No. 2, 2004, pp. 547-559. doi:10.1111/j.1365-2966.2004.07520.x

[22] R. M. Humphreys, "Studies of Luminous Stars in Nearby Galaxies. I. Supergiants and O Stars in the Milky Way," The Astrophysical Journal Supplement, Vol. 38, 1978, pp. 309-350. doi:10.1086/190559

[23] C. D. Garmany and R. E. Stencel, "Galactic OB Associations in the Northern Milky Way Galaxy. I-Longitudes $55^{\circ}$ to $150^{\circ}, "$ Astronomy and Astrophysics Supplement
Series, Vol. 94, No. 2, 1992, pp. 211-244.

[24] H.-T. Lee and J. Lim, "On the Formation of Perseus OB1 at High Galactic Latitudes," The Astrophysical Journal, Vol. 679, No. 2, 2008, pp. 1352-1363. doi: $10.1086 / 587801$

[25] J.-C. Mermilliod and E. Paunzen, "Analysing the Database for Stars in Open Clusters. I. General Methods and Description of the Data," Astronomy and Astrophysics, Vol. 410, No. 2, 2003, pp. 511-518. doi:10.1051/0004-6361:20031112

[26] D. Russeil, "Star-Forming Complexes and the Spiral Structure of Our Galaxy," Astronomy and Astrophysics, Vol. 397, 2003, pp. 133-146. doi:10.1051/0004-6361:20021504

[27] W. S. Dias, B. S. Alessi, A. Moitinho and J. R. Lépine, "New Catalogue of Optically Visible Open Clusters and Candidates," EAS Publications Series, Vol. 10, 2003, p. 195. doi:10.1051/eas:2003148 